\title{
Reaction Kinetics in Polymer Melts
}

\section{BEN O'SHAUGHNESSY ${ }^{1}$ and DIMITRIOS VAVYLONIS ${ }^{2}$}

\author{
${ }^{1}$ Department of Chemical Engineering \\ Columbia University \\ 500 West 120th Street \\ New York, NY 10027, USA \\ e-mail: bo8@columbia.edu \\ ${ }^{2}$ Department of Physics \\ Columbia University \\ 538 West 120th Street \\ New York, NY 10027, USA \\ e-mail: dvav@phys.columbia.edu
}




\section{ABSTRACT}

We study the reaction kinetics of end-functionalized polymer chains dispersed in an unreactive polymer melt. Starting from an infinite hierarchy of coupled equations for many-chain correlation functions, a closed equation is derived for the 2 nd order rate constant $k$ after postulating simple physical bounds. Our results generalize previous 2-chain treatments (valid in dilute reactants limit) by Doi [1], de Gennes [2], and Friedman and O'Shaughnessy [3], to arbitrary initial reactive group density $n_{0}$ and local chemical reactivity $Q$. Simple mean field (MF) kinetics apply at short times, $k \sim Q$. For high $Q$, a transition occurs to diffusioncontrolled (DC) kinetics with $k \approx x_{t}^{3} / t$ (where $x_{t}$ is rms monomer displacement in time $t$ ) leading to a density decay $n_{t} \approx n_{0}-n_{0}^{2} x_{t}^{3}$. If $n_{0}$ exceeds the chain overlap threshold, this behavior is followed by a regime where $n_{t} \approx 1 / x_{t}^{3}$ during which $k$ has the same power law dependence in time, $k \approx x_{t}^{3} / t$, but possibly different numerical coefficient. For unentangled melts this gives $n_{t} \sim t^{-3 / 4}$ while for entangled cases one or more of the successive regimes $n_{t} \sim t^{-3 / 4}, t^{-3 / 8}$ and $t^{-3 / 4}$ may be realized depending on the magnitudes of $Q$ and $n_{0}$. Kinetics at times longer than the longest polymer relaxation time $\tau$ are always MF. If a DC regime has developed before $\tau$ then the long time rate constant is $k \approx R^{3} / \tau$ where $R$ is the coil radius. We propose measuring the above kinetics in a model experiment where radical end groups are generated by photolysis.

PACS numbers: 82.35.+t, 05.40.+j, 05.70.Ln 


\section{Introduction}

The study of polymer-polymer reaction kinetics is a fundamental problem in polymer science. Such reactions occur in many technologically important processes such as vulcanization of rubbers and free radical polymerization [4]. Compared to analogous reactions between small molecules, polymer reaction kinetics are novel in that they reflect static and dynamic properties of the polymer chains which are host to the reacting groups. Theoretical studies predict that rate constants depend both on the degree of polymerization and time [1,2,5]. Model experiments [6,7] and numerical simulations [8,9] testing these laws thus offer a way to probe fundamentals of polymer dynamics. In this paper we focus on reactions between end-functionalized chains in a polymer melt. So far, no systematic experiment exists measuring rate constants in a melt as a function of molecular weight and time. It is our aim to motivate such model experiments by developing a complete theoretical picture for such reactions for direct comparison with experiment.

The situation we analyze is illustrated in fig. 1. We imagine that at $t=0$ a certain fraction of the chains randomly and uniformly distributed in a monodisperse polymer melt carry chemically reactive end-groups. Experimentally this can be realized for example by attaching photocleavable groups to the ends of a certain fraction of the melt chains [10]. A laser pulse then cleaves these groups into radical pairs (fig. 2), one of which is a small molecule and the other of which is attached to the end of the chain (a macroradical) [11]. It was shown in ref. [10] that after a transient the small radicals disappear, leaving behind a fraction of order unity of the macroradicals. The reaction kinetics of these kinetically isolated macroradicals will then follow the kinetics developed in the following.

Anticipating second order kinetics, our aim is to determine the second order time-dependent rate constant $k_{t}$ defined by

$$
\dot{n_{t}} \equiv \frac{d n_{t}}{d t}=-k_{t} n_{t}^{2}
$$

where $n_{t}$ is the number density of reactive groups at time $t$. Now for the usual case of reactions between small molecules, $k_{t}$ is independent of time. This is so because small molecules obey simple Fickian diffusion, $x_{t} \sim t^{1 / 2}$, where $x_{t}$ is the rms displacement in time $t$. This is a very dilute exploration of space (the exploration volume $x_{t}^{3}$ increases faster than $t$ ) and diffusion is fast enough to smooth out any reaction-induced correlations [2, 12, Thus reactant distribution remains uniform and random at all times, the number of pairs per unit volume which are in contact at time $t$ is therefore $a^{3} n_{t}^{2}$, and hence $\dot{n}_{t} \approx Q a^{3} n_{t}^{2}$ where the local reactivity $Q$ measures probability of reaction per unit time when in contact. This implies a time-independent rate constant $k \approx Q a^{3}$. For "infinitely" reactive groups for which $Q$ is effectively equal to the rate at which diffusion brings apart a pair of molecules in contact, i. e. $Q \approx 1 / t_{a}$ where $t_{a}$ is the reactant relaxation time, this leads to the well known Smoluchowski [13] rate constant $k \approx a^{3} / t_{a} \approx D_{\text {small }} a$ where $D_{\text {small }} \approx a^{2} / t_{a}$ is the small molecule self-diffusivity.

Interpolymeric reaction kinetics are more complicated. For times shorter than the longest polymer relaxation time $\tau$, corresponding to a diffusion distance of order the coil radius $R$, reactive groups attached to polymer chains explore space in a dense way: The dynamical exponent $z$, defined by $x_{t} \sim t^{1 / z}$, is 4 or 8 depending on the degree of entanglement and time [14.15]; thus the exploration volume $x_{t}^{3}$ is increasing more slowly than $t$ and the number of times reactive groups visit points within their exploration volumes is an increasing function of time. This was clearly pointed out by de Gennes [2] who differentiated between "compact" 
exploration when $z$ is greater than the dimensionality of space $d$ and "non-compact" for $z<d$.

Now in the limit in which reactants are very dilute, $n_{0} R^{3} \ll 1$, the probability that by time $\tau$ a reactive group's exploration volume contains a second reactive group is very small. Hence for $t<\tau$ reactions are due to the few isolated pairs which happened to be initially within diffusive range, i. e. the members of which were initially within $x_{t}$ of one another. Due to the compact exploration, for times greater than $t$ the number of collisions between such a pair grows without bound. Therefore for "infinitely" reactive groups $\left(Q t_{a} \approx 1\right)$ reaction is almost certain by $t$. Thus a depletion hole of size $x_{t}$ starts to grow in the 2-body correlation function. The number of reacted pairs per unit volume is $x_{t}^{3} n_{0}^{2}$, which when differentiated with respect to time implies a short time time-dependent rate constant $k_{t} \approx x_{t}^{3} / t$. Diffusion dynamics change at $\tau$, since center of gravity Fickian diffusion of the whole chain takes over, $x_{t} \sim t^{1 / 2}$. The hole in the 2-body correlation function stops growing and the situation is as in the small molecule infinite reactivity case, but now with an effective capture radius $R$ reflecting the short time compact dynamics. Thus

$$
k_{t} \approx\left\{\begin{array}{ll}
x_{t}^{3} / t & (t<\tau) \\
R^{3} / \tau & (t>\tau)
\end{array} \quad\left(Q t_{a}=1, n_{0} R^{3} \ll 1\right) .\right.
$$

The $t>\tau$ expression for $k$ was first obtained by Doi [16, 1] for unentangled melts and was extended by de Gennes [2] to $t<\tau$ and entangled cases. Friedman and O'Shaughnessy [3] derived $k$ from first principles using renormalization group calculations.

The generalization of eq. (2) to the concentrated regime, $n_{0} R^{3} \gg 1$, is far less obvious and is one of the main objectives of this paper. In this case the timescale by which a reactive group will almost certainly contain another group within its exploration volume occurs during the compact regime, $t<\tau$. During this regime one can no longer assign reactions to pairs since a given group has the opportunity to react with more than one partner. A proper study of the reaction kinetics requires a full many-body treatment. Such a manybody formalism involves an infinite hierarchy of coupled dynamical equations for correlation functions of different orders [17,18]. Other than renormalization group analyses [19, 20, this complication is typically resolved by decoupling approximations expressing higher order correlations in terms of lower order correlation functions [21. In our approach we are able to derive closed equations after assuming much less restrictive bounds on the magnitude of correlation functions. We show that the implicit assumption of ref. 2, namely that eq. (2) is also valid in the concentrated regime, is correct.

Our study is also general in terms of the local reactivity $Q$. This is very important experimentally, since typical chemical reactivities are extremely small [22, 23], $Q t_{a} \lesssim 10^{-6}$, with the exception of radical species which are very close to being infinitely reactive, $Q t_{a} \approx 1$ [24.

In the following section we present the many-body formalism describing polymer-polymer reaction kinetics and introduce our assumptions on the magnitude of correlation functions. This results in a closed equation for $k$. We solve this equation in section 3 in terms of $x_{t}$ and identify sequences of kinetic regimes depending on the values of $Q$ and $n_{0}$. We apply these general results to the case of unentangled and entangled melts in sections 4 and 5 , respectively. Results are presented in the form of a "phase-diagram" in the $Q-n_{0}$ plane, different regions of which correspond to different kinetic sequences. We conclude with a discussion of our results in section 6 . 


\section{Solution for Rate Constant $k_{t}$}

Consider the situation illustrated in fig. 1 in which reactive ends are initially randomly distributed with density $n_{0}$. Reactions commence at $t=0$. Let us define $\rho_{t}\left(\mathbf{r}_{1}, \mathbf{r}_{2}\right)$ to be the 2 -body correlation function of reactive chain ends located at $\mathbf{r}_{1}, \mathbf{r}_{2}$. Translational invariance implies $\rho_{t}$ is a function of $\mathbf{r}_{1}-\mathbf{r}_{2}$ only. Now the reaction rate per unit volume at point $\mathbf{r}$ is proportional to the number density of pairs in contact at $\mathbf{r}, a^{3} \rho(\mathbf{r}, \mathbf{r})$, multiplied by the local reactivity $Q$. Here $a$ is the monomer size. Using the fact that translational invariance implies the average density $n_{t}$ is independent of $\mathbf{r}$ one has

$$
\dot{n}_{t}=-\lambda \rho_{t}(0,0), \quad \lambda \equiv Q a^{3} .
$$

Thus the calculation of the reaction rate requires evaluation of the 2-body correlation function. A rigorous dynamical equation for $\rho_{t}$ in the case of reactions between small molecules has been derived by Doi in ref. 18. In the polymer reaction problem considered here, in order to derive a closed relationship for $\rho_{t}$ in terms of the degrees of freedom specifying the location of the reactive ends only, one must first average out the degrees of freedom specifying the locations of the other monomers; this is non-trivial and requires renormalization group (RG) methods. However, RG studies of 2-body bulk polymer reaction kinetics [25]:3] indicate that the basic physics is completely captured by the approximate closing of the system in terms of the coordinates of reactive groups: correct scaling behaviors are obtained, only the prefactors being unreliable. Making the approximation of closure in terms of reactive degrees of freedom, then the $\rho_{t}$ dynamics involves the 3 -body reactive groups correlation function $\rho_{t}^{(3)}\left(\mathbf{r}_{1}, \mathbf{r}_{2}, \mathbf{r}_{3}\right)$ 18,26:

$$
\begin{aligned}
\rho_{t}\left(\mathbf{r}_{1}, \mathbf{r}_{2}\right) & =n_{0}^{2}-\lambda \int d \mathbf{r}_{1}^{\prime} d \mathbf{r}_{2}^{\prime} \int_{0}^{t} d t^{\prime} G_{t-t^{\prime}}\left(\mathbf{r}_{1}, \mathbf{r}_{2}, \mathbf{r}_{1}^{\prime}, \mathbf{r}_{2}^{\prime}\right) \rho_{t^{\prime}}\left(\mathbf{r}_{1}^{\prime}, \mathbf{r}_{2}^{\prime}\right) \delta\left(\mathbf{r}_{1}^{\prime}-\mathbf{r}_{2}^{\prime}\right) \\
& -\lambda \int d \mathbf{r}_{1}^{\prime} d \mathbf{r}_{2}^{\prime} d \mathbf{r}_{3}^{\prime} \int_{0}^{t} d t^{\prime} G_{t-t^{\prime}}\left(\mathbf{r}_{1}, \mathbf{r}_{2}, \mathbf{r}_{1}^{\prime}, \mathbf{r}_{2}^{\prime}\right) \rho_{t^{\prime}}^{(3)}\left(\mathbf{r}_{1}^{\prime}, \mathbf{r}_{2}^{\prime}, \mathbf{r}_{3}^{\prime}\right)\left\{\delta\left(\mathbf{r}_{1}^{\prime}-\mathbf{r}_{3}^{\prime}\right)+\delta\left(\mathbf{r}_{2}^{\prime}-\mathbf{r}_{3}^{\prime}\right)\right\},
\end{aligned}
$$

where $G_{t}\left(\mathbf{r}_{1}, \mathbf{r}_{2}, \mathbf{r}_{1}^{\prime}, \mathbf{r}_{2}^{\prime}\right)$ is the equilibrium chain end propagator giving the net weighting for two ends to arrive at $\mathbf{r}_{1}, \mathbf{r}_{2}$ given starting points $\mathbf{r}_{1}^{\prime}, \mathbf{r}_{2}^{\prime}$ in the absence of reactions. This is a well known object. The sink terms on the right hand side of eq. (4) (illustrated in fig. 3) describe the three ways in which reactions diminish $\rho_{t}$ from its initial value $n_{0}^{2}$. The first two-body sink term subtracts off pairs which failed to reach $\mathbf{r}_{1}, \mathbf{r}_{2}$ because their members reacted with one another at $\mathbf{r}_{1}^{\prime}$ at time $t^{\prime}$. The remaining two sink terms subtract off pairs which would be at $\mathbf{r}_{1}, \mathbf{r}_{2}$ but only one member of which reacted at time $t^{\prime}$ at location $\mathbf{r}_{3}^{\prime}$. Such a reaction involves a third chain, weighted by the appropriate 3 -chain correlation function. These are many-chain terms; were they absent, one would have a closed 2-chain system. In refs. 11 and 2 these terms were omitted. As explained in the introduction one expects such a 2 -body approach to be valid in the dilute limit. In eq. (4) we used a $\delta$ function as a reactive sink. This is a coarse-grained description of the reaction process over a scale of order the monomer size $a$.

Notice that eq. (4) is not closed in terms of $\rho_{t}$ since it involves the unknown $\rho_{t}^{(3)}$. It is in fact impossible to write a closed exact equation for $\rho_{t}$ since correlation functions of all orders are coupled in an infinite hierarchy of dynamical equations [18, 17]. This complication which arises in all many-body reacting systems is typically resolved by approximating 3body correlations in terms of lower order correlation functions [21]. In this study we are able 
to write a closed equation for $\rho_{t}$ after assuming less restrictive bounds on the magnitude of correlation functions. To explore the constraints imposed by these bounds we first transform eq. (4) to an integral expression for the function $q_{t}(\mathbf{r}, 0) \equiv \rho_{t}(\mathbf{r}, 0) / n_{t}^{2}$. Notice that eq. (1) then implies $k_{t}=\lambda q_{t}(0,0)$. It is demonstrated in appendix $\mathrm{A}$ that after making this change of variables, one has

$$
\begin{aligned}
q_{t}(\mathbf{r}, 0) & =1-\int d \mathbf{r}_{1}^{\prime} \int_{0}^{t} d t^{\prime} G_{t-t^{\prime}}\left(\mathbf{r}, 0, \mathbf{r}_{1}^{\prime}, \mathbf{r}_{1}^{\prime}\right) k_{t^{\prime}} \\
& +2 \int d \mathbf{r}_{1}^{\prime} d \mathbf{r}_{2}^{\prime} \int_{0}^{t} d t^{\prime} G_{t-t^{\prime}}\left(\mathbf{r}, 0, \mathbf{r}_{1}^{\prime}+\mathbf{r}_{2}^{\prime}, \mathbf{r}_{2}^{\prime}\right) k_{t^{\prime}}\left\{\rho_{t^{\prime}}\left(\mathbf{r}_{1}^{\prime} \mid 0\right)-\rho_{t^{\prime}}^{(3)}\left(\mathbf{r}_{1}^{\prime} \mid 0,0\right)\right\}
\end{aligned}
$$

where $\rho_{t}(\mathbf{r} \mid 0)$ and $\rho_{t}^{(3)}(\mathbf{r} \mid 0,0)$ are the conditional reactive chain end densities at $\mathbf{r}$, given one and two reactive ends, respectively, at the origin:

$$
\rho_{t}(\mathbf{r} \mid 0) \equiv \frac{\rho_{t}(\mathbf{r}, 0)}{n_{t}}, \quad \rho_{t}^{(3)}(\mathbf{r} \mid 0,0) \equiv \frac{\rho_{t}^{(3)}(\mathbf{r}, 0,0)}{\rho_{t}(0,0)}
$$

Eq. (5) can be reexpressed in terms of the propagator $G_{t}^{\mathrm{sep}}\left(\mathbf{r}_{1}, \mathbf{r}_{2}\right) \equiv \int d \mathbf{r}_{2}^{\prime} G_{t}\left(\mathbf{r}_{1}^{\prime}, \mathbf{r}_{1}^{\prime}+\right.$ $\mathbf{r}_{1}, \mathbf{r}_{2}^{\prime}, \mathbf{r}_{2}^{\prime}+\mathbf{r}_{2}$ ), namely the probability density two chains ends are separated by $\mathbf{r}_{1}$ at time $t$ given initial separation $\mathbf{r}_{2}$. Thus eq. (5) becomes

$$
q_{t}(\mathbf{r}, 0)=1-\int_{0}^{t} d t^{\prime} G_{t-t^{\prime}}^{\mathrm{sep}}(\mathbf{r}, 0) k_{t^{\prime}}+\varphi_{t}(\mathbf{r})
$$

where

$$
\varphi_{t}(\mathbf{r}) \equiv \int_{0}^{t} d t^{\prime} \int d \mathbf{r}^{\prime} G_{t-t^{\prime}}^{\mathrm{sep}}\left(\mathbf{r}, \mathbf{r}^{\prime}\right) k_{t^{\prime}} \mu_{t^{\prime}}\left(\mathbf{r}^{\prime}\right), \quad \mu_{t}(\mathbf{r}) \equiv 2\left\{\rho_{t}(\mathbf{r} \mid 0)-\rho_{t}^{(3)}(\mathbf{r} \mid 0,0)\right\}
$$

We want to solve eq. (77) for $k_{t}$ or equivalently for $q_{t}(0,0)$. This requires information on the properties of $\varphi_{t}(0)$ which in turn involves 2 -body and 3 -body conditional densities. Now we make the assumption that the more reactive groups placed at the origin, the lower the conditional density. Chemical reactivity can only induce anticorrelations. Thus:

$$
\rho_{t}^{(3)}(\mathbf{r} \mid 0,0) \leq \rho_{t}(\mathbf{r} \mid 0) \leq n_{t} \quad \text { (assumption) } .
$$

Eq. (9) immediately implies the following constraint on $\mu$ (see eq. (8))

$$
0 \leq \mu_{t}(\mathbf{r}) \leq 2 n_{t}
$$

Eq. (9) also implies that $q_{t} \leq 1$. Thus the magnitude of the second term on the rhs of eq. (7) must exceed that of the $3 \mathrm{rd}$ term, $\int_{0}^{t} d t^{\prime} G_{t-t^{\prime}}^{\mathrm{sep}}(\mathbf{r}, 0) k_{t^{\prime}} \geq \varphi_{t}(\mathbf{r})$. This is the full inequality which must be obeyed by the function $\mu_{t^{\prime}}\left(\mathbf{r}^{\prime}\right)$ involving the unknown $\rho^{(3)}$. Integrating over $\mathbf{r}$ yields the following less demanding inequality:

$$
\int_{0}^{t} d t^{\prime} k_{t^{\prime}} \geq \int_{0}^{t} d t^{\prime} \int d \mathbf{r}^{\prime} k_{t^{\prime}} \mu_{t^{\prime}}\left(\mathbf{r}^{\prime}\right)
$$


On the strength of the above constraints, eqs. (10) and (111), we will now argue that the solution of eq. (17) for $q_{t}(0,0)$, but with the term $\varphi_{t}(0)$ deleted, gives the correct power law solution for $k_{t}$ to within a constant prefactor. Expressing $q_{t}(0,0)$ in terms of $k_{t}$, setting $\mathbf{r}=0$ and deleting $\varphi_{t}(0)$, eq. (17) becomes

$$
k_{t}=\lambda-\lambda \int_{0}^{t} d t^{\prime} S_{t-t^{\prime}} k_{t^{\prime}}
$$

where we have introduced the return probability $S_{t} \equiv G_{t}^{\text {sep }}(0,0)$.

In appendix B, a self-consistent argument is presented to justify the deletion of $\varphi_{t}(0)$. A summary of this argument is as follows. Now if one accepts eq. (12), then eqs. (12) and (1) lead to a sequence of power law regimes in time, both for $k_{t}$ and $n_{t}$ (these are explicitly obtained in the next sections). Using these solutions in the constraints (eqs. (10) and (11)) and in the expression for $\varphi_{t}(0)$ (eq. (8)), the function $\mu_{t^{\prime}}^{\max }\left(\mathbf{r}^{\prime}\right)$ which maximizes $\varphi_{t}(0)$ (for a given time $t$ ) subject to the constraints is determined. This in turn implies an upper bound on $\varphi_{t}(0)$, namely $\varphi_{t}^{\max }(0)$, according to eq. (8). It is then shown in appendix $\mathrm{B}$ that $\varphi_{t}^{\max }(0) \ll 1$. (Of course, in reality there is one unique value for $\varphi_{t}(0)$, since $\mu_{t^{\prime}}\left(\mathbf{r}^{\prime}\right)$ is a determined function, eq. (8). What has been achieved here is to bound this function given our incomplete knowledge.) Thus $\varphi_{t}(0)$ may be deleted in eq. (7) without error; even prefactors are expected to be correct. There is, however, one exception to this simple state of affairs. During those time regimes where $n_{t} \approx 1 / x_{t}^{3}$ (see next sections) we find $\varphi_{t}^{\max }(0)=A$, where $A$ is a constant of order unity. For these regimes, we are forced to make one further assumption, that $k_{t}$ remains a power law during this regime. In appendix $\mathrm{B}$, it is shown that this implies that the possibility of $\varphi_{t}(0)$ being of order unity may modify the coefficient of $k_{t}$ only, not the power itself.

Thus in the following we simply solve eq. (12). This equation has been derived in ref. 2 starting from a 2-body formalism valid in the dilute reactive species limit as discussed. Here we argue that it is valid for all $n_{0}$. Expressions equivalent to eq. (12) were the starting point in ref. 27 the authors of which pointed out the existence of a $n_{t} \approx 1 / x_{t}^{3}$ regime in polymer reaction kinetics.

Now Laplace transforming $t \rightarrow E$ one immediately solves eq. (12) for $k(E)$ :

$$
k(E)=\frac{\lambda}{E(1+\lambda S(E))},
$$

which is the main result of this section.

\section{Kinetic Regimes and Timescales}

In the previous section we obtained a solution for $k$ in Laplace space. This involves the return probability $S_{t}$. It is easy to show [2] that $S_{t} \approx 1 / x_{t}^{3}$ where $x_{t}$ is rms displacement. In this section by substitution in eq. (13) we obtain solutions for $k$ and $n$ in terms of $x_{t}$. Explicit forms of $x_{t}$ for unentangled and entangled melts are considered in sections 4 and 5 .

For times shorter than the longest polymer relaxation time $\tau$ (corresponding to $E \gg \tau$ ), $x_{t} \sim t^{1 / z}$ with $z=4$ and 8 depending on time and the degree of entanglement 14, 15. Thus $S_{t} \sim t^{-3 / z}$ which implies $S(E) \sim E^{3 / z-1}$ for $1 / E \ll \tau$. For times $t \gg \tau$, center of gravity Fickian diffusion applies, $x_{t} \sim t^{1 / 2}$. Thus $S_{t} \sim t^{-3 / 2}$ and it follows that for $1 / E \gg \tau, S(E)$ approaches its $E=0$ limit, $\int_{0}^{\infty} d t S(t) \approx \tau / R^{3}$ (see refs. 1,2). Thus, as a function of $1 / E$, $S(E)$ increases and then saturates at $\tau$. 
To determine $k_{t}$ we must determine which of the two terms, 1 or $\lambda S(E)$ in eq. (13) is dominant. There are two cases. If $Q$ (or $\lambda$ ) is sufficiently small, $Q<\widehat{Q}$ (see definition below), then $\lambda S(E) \ll 1$ for all $E$ values, implying

$$
k_{t} \approx \lambda, \quad(Q<\widehat{Q}) .
$$

For $Q>\widehat{Q}$ there exists a $1 / E$ value (shorter than $\tau$ ), corresponding to a timescale $t_{2}^{*}$, after which $\lambda S(E) \gg 1$. In this case during the regime $t_{2}^{*} \ll E^{-1} \ll \tau, k(E) \approx 1 / E S(E) \sim E^{-3 / z}$. Thus Laplace inverting eq. (13) we have:

$$
k_{t} \approx\left\{\begin{array}{ll}
\lambda & \left(t \ll t_{2}^{*}\right) \\
x_{t}^{3} / t & \left(t_{2}^{*} \ll t \ll \tau\right) \\
R^{3} / \tau & (t \gg \tau)
\end{array} \quad(Q>\widehat{Q})\right.
$$

The quantities $t_{2}^{*}$ and $\widehat{Q}$ are defined by

$$
\frac{x_{t_{2}^{*}}^{3}}{t_{2}^{*}}=\lambda \quad\left(t_{2}^{*}<\tau\right), \quad \widehat{Q}=\frac{R^{3}}{a^{3} \tau} .
$$

The value of $t_{2}^{*}$ is determined by demanding continuity of $k_{t}$. In Laplace space, $t_{2}^{*}$ corresponds to the $1 / E$ value value at which $\lambda S(E)$ becomes of order unity. Eq. (16) has a solution for $t_{2}^{*}$ only if $Q$ is large enough so that $t_{2}^{*}<\tau$. Thus the condition $t_{2}^{*}=\tau$ defines $\widehat{Q}$ in eq. (16).

Now let us see what eqs. (14) and (15) imply for the decay of $n_{t}$, considering the cases $Q>\widehat{Q}$ and $Q<\widehat{Q}$ in turn.

1. Case $Q>\widehat{Q}$. The solution of eq. (四) is $n_{t}=n_{0} /\left(1+n_{0} \int_{0}^{t} k_{t^{\prime}} d t^{\prime}\right)$. Thus using eq. (15) one has

$$
\frac{n_{t}}{n_{0}} \approx\left\{\begin{array}{ll}
1 /\left(1+t / t_{m}^{*}\right) & \left(t \ll t_{2}^{*}\right) \\
1 /\left(1+n_{0} x_{t}^{3}\right) & \left(t_{2}^{*} \ll t \ll \tau\right) \\
1 /\left(1+t / t_{m, R}^{*}\right) & (t \gg \tau)
\end{array} \quad(Q>\widehat{Q})\right.
$$

We define the timescales $t_{m}^{*}, t_{m, R}^{*}$ and $t_{l}$ by

$$
t_{m}^{*} \equiv \frac{1}{\lambda n_{0}}, \quad t_{m, R}^{*} \equiv \frac{\tau}{n_{0} R^{3}}, \quad x_{t_{l}}^{3}=\frac{1}{n_{0}} .
$$

Here $t_{l}$ is the time to diffuse a distance of the order of the typical initial separation between reactants. Notice that at a certain timescale the time-dependent term in the denominator of $n_{t}$ in eq. (18) becomes of order unity. This time is one of $t_{m}^{*}, t_{l}$, and $t_{m, R}^{*}$. Different kinetic behavior occurs depending on the ordering of these timescales amongst themselves and with respect to $t_{2}^{*}$ and $\tau$.

Let us consider first the kinetics in the "concentrated" case, $n_{0} R^{3}>1$, i. e. $t_{l}>\tau$. Then the monotonically increasing time-dependent term in the denominator of $n_{t}$ in eq. (17) becomes of order unity before $\tau$. There are two possibilities depending on whether or not $Q$ is large enough such that $t_{2}^{*}$ is smaller than $t_{l}$ (notice that $t_{l}$ is independent of $Q$ ). Now if indeed $t_{2}^{*}<t_{l}$ one has

$$
n_{t} \approx n_{0}-\lambda n_{0}^{2} t \stackrel{t_{2}^{*}}{\longrightarrow} n_{0}-n_{0}^{2} x_{t}^{3} \stackrel{t_{l}}{\longrightarrow} \frac{1}{x_{t}^{3}} \stackrel{\tau}{\longrightarrow} \frac{1}{R^{3} t / \tau} \quad\left(t_{2}^{*}<t_{l}<\tau, \mathrm{S}^{\text {conc }}\right)
$$

We use the symbol $\mathrm{S}^{\text {conc }}$ for "strong concentrated" since eq. (19) applies for high $Q$ and $n_{0}$ values (see phase-diagrams of figs. 4, 5). Notice that we do not need to specify $Q>\widehat{Q}$ in eq. 
(19) Since only for such $Q$ values is $t_{2}^{*}<\tau$. If on the other hand $t_{2}^{*}>t_{l}$, one similarly has

$$
n_{t} \approx n_{0}-\lambda n_{0}^{2} t \stackrel{t_{m}^{*}}{\longrightarrow} \frac{1}{\lambda t} \stackrel{t_{2}^{*}}{\longrightarrow} \frac{1}{x_{t}^{3}} \stackrel{\tau}{\longrightarrow} \frac{1}{R^{3} t / \tau} \quad\left(Q>\widehat{Q}, t_{l}<t_{2}^{*}, \mathrm{~W}\right)
$$

where W stands for "weak" since these systems are less reactive when compared to the strong concentrated regime (see figs. 1 and 5). Notice that we do not need to specify $t_{l}>\tau$ in eq. (2Q), since this is implied by $Q>\widehat{Q}$ (or equivalently $t_{2}^{*}<\tau$ ) and $t_{l}<t_{2}^{*}$.

Now in the the "dilute" case, $n_{0} R^{3}<1$, the time-dependent term in the denominator of $n_{t}$ in eq. (17) becomes of order unity for $t>\tau$ :

$$
n_{t} \approx n_{0}-\lambda n_{0}^{2} t \stackrel{t_{2}^{*}}{\longrightarrow} n_{0}-n_{0}^{2} x_{t}^{3} \stackrel{\tau}{\longrightarrow} n_{0}-n_{0}^{2} R^{3} \frac{t}{\tau} \stackrel{t_{m, R}^{*}}{\longrightarrow} \frac{1}{R^{3} t / \tau} \quad\left(Q>\widehat{Q}, t_{l}>\tau, \mathrm{S}^{\mathrm{dil}}\right) .
$$

Here $\mathrm{S}^{\text {dil }}$ stands for "strong dilute."

2. Case $Q<\widehat{Q}$. Finally let us examine the $Q<\widehat{Q}$ case. Substituting eq. (14) in (11) one immediately has:

$$
n_{t} \approx n_{0}-\lambda n_{0}^{2} t \stackrel{t_{m}^{*}}{\longrightarrow} \frac{1}{\lambda t} \quad(Q<\widehat{Q}, \mathrm{WW})
$$

We use the symbol WW (very weak) to refer to systems belonging to this class which correspond to the lowest $Q$ values in which mean field kinetics are always applicable.

We have thus identified 4 distinct behaviors, eqs. (19)-(22), for the decay of $n_{t}$, corresponding to 4 regions in the $Q-n_{0}$ plane. In the following two sections we explicitly plot these regions for both unentangled and entangled melts after giving expressions for $x_{t}$ as dictated by the Rouse and reptation model, respectively.

\section{Application to Unentangled Melts}

The dynamics of polymers in an unentangled melt, i. e. with degree of polymerization $N$ shorter than the entanglement threshold $N_{\mathrm{e}}$, are well known to obey Rouse dynamics [15, 14]:

$$
x_{t} \approx\left\{\begin{array}{lll}
a\left(t / t_{a}\right)^{1 / 4} & (t<\tau) \\
R(t / \tau)^{1 / 2} & (t>\tau)
\end{array} \quad \frac{\tau}{t_{a}}=N^{2}, \quad \frac{R}{a}=N^{1 / 2}\right.
$$

Thus Rouse dynamics is characterized by compact $(z=4)$ short time behavior, followed by noncompact Fickian diffusion $(z=2)$.

By substitution of eq. (23) in eqs. (16) and (18) we obtain explicit expressions for $t_{2}^{*}$ and $t_{l}$ :

$$
t_{2}^{*}=t_{a} /\left(Q t_{a}\right)^{4} \quad(Q>\widehat{Q}), \quad t_{l}=t_{a} /\left(a^{3} n_{0}\right)^{4 / 3} \quad\left(n_{0} R^{3}>1\right)
$$

(the timescale $t_{l}$ is only relevant to the kinetics when $n_{0} R^{3}>1$ ). Timescales $t_{m}^{*}, t_{m, R}^{*}$ are already given explicitly in eq. (18). Using these expressions, in fig. 国 the lines $t_{2}^{*}=\tau, t_{l}=\tau$, and $t_{l}=t_{2}^{*}$ have been drawn in the $Q-n_{0}$ plane. Certain sections of these lines are omitted, in those regions where they are irrelevant. These lines define four distinct regions. It is straightforward to verify that each one of these corresponds to one of the kinetic regimes defined in eqs. (19)-(22) as indicated in the figure. 
Notice that the condition $t_{l}=t_{2}^{*}$ also implies $t_{l}=t_{2}^{*}=t_{m}^{*}$, as one can easily verify using eqs. (16) and (18). In the $Q-n_{0}$ plane, the $t_{l}=t_{2}^{*}=t_{m}^{*}$ line separating the $\mathrm{S}^{\text {conc }}$ from the $\mathrm{W}$ region thus defines a density-dependent reactivity $Q^{*}$ :

$$
Q^{*} t_{a}=\left(n_{0} a^{3}\right)^{1 / 3}
$$

indicated in fig. 4 .

Perhaps the most interesting feature of the unentangled case is the $n_{t} \sim t^{-3 / 4}$ decay of $n_{t}$ during the $n_{t} \approx 1 / x_{t}^{d}$ regime in regions $\mathrm{S}^{\text {conc }}, \mathrm{W}$.

\section{Application to Entangled Melts}

In the case of very long chains, $N>N_{\mathrm{e}}$, polymer motion is affected by entanglements. In the reptation model [15,14] entanglements are assumed to inhibit lateral chain motion on the scale of the "tube" of diameter $r_{\mathrm{e}}=N_{\mathrm{e}}^{1 / 2} a$, corresponding to a portion of chain comprising $N_{\mathrm{e}}$ units. For times shorter than the diffusion time $t_{\mathrm{e}}=N_{\mathrm{e}}^{2} t_{a}$ to distance $r_{\mathrm{e}}$, monomers do not feel the tube and obey Rouse-like $t^{1 / 4}$ dynamics as in unentangled melts. For $t>t_{\mathrm{e}}$, the chain diffuses curvilinearly up and down the tube in 1-dimensional $t^{1 / 4}$ Rouse motion. During these "breathing modes," monomer rms displacement in space increases as $t^{1 / 8}$, since the tube is itself a random walk. The chain relaxes its configuration relative to the tube by time $t_{\mathrm{b}}=N^{2} t_{a}$, corresponding to monomer diffusion distance $r_{\mathrm{b}}=r_{\mathrm{e}}\left(N / N_{\mathrm{e}}\right)^{1 / 4}$. For longer times, coherent diffusion along the tube gives rise to $t^{1 / 4} \mathrm{rms}$ monomer displacement. This regime persists until the longest polymer relaxation or "reptation" time, $\tau=\left(R / r_{\mathrm{b}}\right)^{4} t_{\mathrm{b}}=$ $\left(N^{3} / N_{\mathrm{e}}\right) t_{a}$, by which time the chain has completely diffused out of its initial tube into a new and uncorrelated one (here $R=N^{1 / 2} a$ ). The process then repeats itself indefinitely, corresponding to long time Fickian center of gravity motion, $x_{t}=R(t / \tau)^{1 / 2}$. In summary,

$$
x_{t} \approx \begin{cases}a\left(t / t_{a}\right)^{1 / 4} & \left(t<t_{\mathrm{e}} \equiv N_{\mathrm{e}}^{2} t_{a}\right) \\ r_{\mathrm{e}}\left(t / t_{\mathrm{e}}\right)^{1 / 8} & \left(t_{\mathrm{e}}<t<t_{\mathrm{b}} \equiv N^{2} t_{a}\right) \\ r_{\mathrm{b}}\left(t / t_{\mathrm{b}}\right)^{1 / 4} & \left(t_{\mathrm{b}}<t<\tau \equiv\left(R / r_{\mathrm{b}}\right)^{4}=N^{3} t_{a} / N_{\mathrm{e}}\right) \\ R(t / \tau)^{1 / 2} & (t>\tau)\end{cases}
$$

Thus, there are 3 compact regimes with a sequence of dynamical exponents $z=4,8,4$, followed by noncompact $z=2$ exploration.

Substituting eq. (26) in eq. (16) one obtains

$$
t_{2}^{*} \approx \begin{cases}t_{a}\left(Q t_{a}\right)^{-4} & \left(Q>r_{\mathrm{e}}^{3} /\left(t_{\mathrm{e}} a^{3}\right)\right) \\ t_{\mathrm{e}}\left(Q t_{\mathrm{e}} a^{3} / r_{\mathrm{e}}^{3}\right)^{-8 / 5} & \left(r_{\mathrm{b}}^{3} /\left(t_{\mathrm{b}} a^{3}\right)<Q<r_{\mathrm{e}}^{3} /\left(t_{\mathrm{e}} a^{3}\right)\right) \\ t_{\mathrm{b}}\left(Q t_{\mathrm{b}} a^{3} / r_{\mathrm{b}}^{3}\right)^{-4} & \left(\widehat{Q}<Q<r_{\mathrm{b}}^{3} /\left(t_{\mathrm{b}} a^{3}\right)\right)\end{cases}
$$

Notice that, unlike the unentangled case, the appropriate formula for $t_{2}^{*}$ is now dependent on the dynamical regime during which it happens to occur. This is determined by $Q$. Similar remarks apply to $t_{l}$, expressions for which depend on $n_{0}$. Eq. (26) in eq. (18) leads to

$$
t_{l} \approx \begin{cases}t_{a}\left(n_{0} a^{3}\right)^{4 / 3} & \left(n_{0}>r_{\mathrm{e}}^{-3}\right) \\ t_{\mathrm{e}}\left(n_{0} r_{\mathrm{e}}^{3}\right)^{8 / 3} & \left(r_{\mathrm{e}}^{-3}>n_{0}>r_{\mathrm{b}}^{-3}\right) \\ t_{\mathrm{b}}\left(n_{0} r_{\mathrm{b}}^{3}\right)^{4 / 3} & \left(r_{\mathrm{b}}^{-3}>n_{0}>R^{-3}\right)\end{cases}
$$


Once again the condition $t_{l}=t_{2}^{*}=t_{m}^{*}$ defines a density-dependent reactivity $Q^{*}$ :

$$
Q^{*}= \begin{cases}\left(n_{0} a^{3}\right)^{1 / 3} / t_{a} & \left(n_{0}>r_{\mathrm{e}}^{-3}\right) \\ \left(n_{0} r_{\mathrm{e}}^{3}\right)^{8 / 3} /\left(t_{\mathrm{e}} n_{0} a^{3}\right) & \left(r_{\mathrm{e}}^{-3}>n_{0}>r_{\mathrm{b}}^{-3}\right) \\ \left(n_{0} r_{\mathrm{b}}^{3}\right)^{4 / 3} /\left(t_{\mathrm{b}} n_{0} a^{3}\right) & \left(r_{\mathrm{b}}^{-3}>n_{0}>R^{-3}\right)\end{cases}
$$

Similarly to the unentangled case, the phase diagram of fig. 5 is constructed. Now the three $Q>\widehat{Q}$ regions develop fine structure. In each of these subregions, crossovers in $n_{t}$ behavior occur during different short time compact regimes.

How does one use this phase diagram to determine the reaction kinetics for a given system? The system's $Q$ and $n_{0}$ values define a point in the diagram. Depending on which of the 4 regions, $\mathrm{S}^{\text {conc }}, \mathrm{S}^{\mathrm{dil}}$, W, WW, this point happens to belong to, the reaction kinetics are then given by the appropriate member of the 4 equations (19)-(22). But in these expressions for reaction kinetics timescales $t_{2}^{*}$ and $t_{l}$ may appear. The appropriate formulae for these timescales are determined by the fine structure location of the point within the region (eqs. (24) and (28)). The other timescales, $t_{m}^{*}, t_{m, R}^{*}$, if relevant, are given by eq. (18).

For example, let us consider point $\alpha$ in fig. 5 belonging to region $\mathrm{S}^{\text {conc }}$. According to eq. (19) the relevant timescales (in addition to $\tau$ ) are $t_{2}^{*}$ and $t_{l}$. Fig. 5 then indicates $t_{2}^{*}<t_{\mathrm{e}}$ and $t_{\mathrm{e}}<t_{l}<t_{\mathrm{b}}$. Thus the $n_{0}-\lambda n_{0}^{2} t$ regime of eq. (19) occurs before $t_{\mathrm{e}}$, followed by $n_{0}-n_{0}^{2} x_{t}^{3}$ which occurs during the latter part of the first $x_{t} \sim t^{1 / 4}$ regime and the earlier part of of the $x_{t} \sim t^{1 / 8}$ regime. The crossover to $n_{t} \approx 1 / x_{t}^{3}$ occurs during the $t^{1 / 8}$ regime, lasting up to $\tau$. During these times $n_{t} \sim t^{-3 / 8}$ followed by $n_{t} \sim t^{-3 / 4}$.

\section{Conclusions}

This study has addressed the kinetics of reactions between end-functionalized polymer chains diffusing in an unreactive polymer melt matrix, as a function of initial reactant density $n_{0}$ and chemical reactivity $Q$. Results were presented in the form of a "phase diagram" in the $Q-n_{0}$ plane.

At short times, simple mean field (MF) kinetics apply: the rate constant $k \approx Q a^{3}$ is independent of time. These kinetics give rise to a density decay $n_{t} \approx n_{0}-n_{0} t / t_{m}^{*}$. In regions $\mathrm{W}$ and WW of the $Q-n_{0}$ plane (figs. (1) and 5) these MF kinetics persist for times greater than $t_{m}^{*}$, leading to $n_{t} \sim 1 / Q t$. Physically, $t_{m}^{*}$ as defined in eq. (18) is the timescale after which a reactive group is very likely to have reacted with the "mean reactive field" supplied by all other reactants in the system.

A transition to diffusion-controlled (DC) kinetics occurs for sufficiently reactive groups, $Q>\widehat{Q}$. This is a result of compact exploration of space at times shorter than the longest chain relaxation time $\tau$. Thus a timescale $t_{2}^{*}$ exists after which any pair which was initially within diffusive range (i. e. the exploration volumes of its members are overlapping by $t$ ) is bound to react. If $t_{2}^{*}$ occurs at times shorter than the time $t_{l}$ needed for a reactive end to diffuse a distance of order the typical separation between reactive groups (regions $\mathrm{S}^{\text {conc }}, \mathrm{S}^{\text {dil }}$ of figs. A and 5), then the depletion in the initial density $n_{0}$ is proportional to the number of pairs with initial separation less than $x_{t}: n_{t} \approx n_{0}-n_{0}^{2} x_{t}^{3}$. Equivalently, $k_{t} \approx x_{t}^{3} / t$ [2]. For unentangled melts this leads to $k_{t} \sim t^{-1 / 4}$, while for entangled cases successive regimes $k_{t} \sim t^{-1 / 4}, t^{-5 / 8}$ and $t^{-1 / 4}$ may exist depending on $Q, n_{0}$.

In regions $\mathrm{S}^{\text {conc }}$ and $\mathrm{W}$ kinetics are diffusion-controlled for times longer than $t_{l}$. During these times the exploration volumes of reactive groups overlap. We find a density decay 
$n_{t} \sim 1 / x_{t}^{3}$ [27. Roughly, this means that only one reactant exists within a region of size comparable to the exploration volume $x_{t}^{3}$; had there been several, they would have reacted. For unentangled melts this implies $n_{t} \sim t^{-3 / 4}$, while an extra $n_{t} \sim t^{-3 / 8}$ regime arises in entangled systems. These DC kinetics are analogous to the anomalous long time decay $n_{t} \approx 1 / x_{t}^{d}$ in the case of small molecule reactions $(A+A \rightarrow \emptyset)$ obeying Fickian dynamics, $x_{t} \sim t^{1 / 2}$, in spatial dimensions below a critical dimension $d_{c}=2$. For arbitrary dynamics, $x_{t} \sim t^{1 / z}$, the critical dimension is $d_{c}=z$; thus $d=3$ is below $d_{c}$ for $t<\tau$ since $z$ is then either 4 or 8 . Notice that the rate constant during these times, $k_{t} \approx x_{t}^{3} / t$, is the same as for short time $\left(t<t_{l}\right)$ DC kinetics (as described above). However there is no obvious reason to expect them to be identical since each one has different physical origins. Indeed, our calculations suggest that the numerical coefficients of these two rate constants are different.

All diffusion-controlled kinetics are truncated at $\tau$ which marks a crossover to Fickian center of gravity polymer diffusion. Thus long time kinetics are always MF. In cases where DC kinetics preceded, the short time kinetics are reflected in a "renormalized" rate constant $k \approx R^{3} / \tau$ [1.2] leading to $n_{t} \approx 1 /\left(R^{3} t / \tau\right)$ at long times.

Experimentally, the most interesting region of the $Q-n_{0}$ plane is $\mathrm{S}^{\text {conc }}$. This region includes the rather peculiar $n_{t} \approx 1 / x_{t}^{3}$ decay. Probing reaction kinetics in this region would require extremely reactive species such as radicals (or electronically excited groups as in photophysical systems [6]) since typical chemical reactivities, $Q t_{a} \lesssim 10^{-6}$, are far below the $Q=\widehat{Q}$ threshold, even for very long chains (see figs. A and fig. 5). One can imagine experimentally probing the $S^{\text {conc }}$ region by photocleaving functional groups attached to polymer chain ends with a laser pulse, generating radical pairs with initial density $n_{0}$ as in fig 2 . Each pair consists of a polymer radical $(\mathrm{P})$ and a monomeric radical $(\mathrm{M})$. An experiment of this type was studied theoretically in refs. 10 and 28 . There are 2 underlying principles: (1) For large $N$, since polymers are much less mobile than monomers, the polymer-polymer reaction constant $k_{P P}$ is much less than the monomer-monomer constant $k_{M M}$. (2) Polymermonomer reactions are dominated by the more mobile monomer [29], i. e. $k_{M P} \approx k_{M M}$ to within a prefactor of order unity. In the following we take $k_{M P}=k_{M M}$ for simplicity. It was shown in refs. 10,28 that after a time $T \approx\left(k_{M M} n_{0}\right)^{-1}$ virtually all $\mathrm{M}$ have reacted, leaving behind a finite fraction of the $\mathrm{P}$ : the polymer radicals are kinetically isolated and the $t>T$ kinetics involve polymer-polymer reactions only (fig. 1). Specifically, after time $T$ a fraction $1 / e$ of the $\mathrm{P}$ remain whilst only a fraction $\epsilon / e$ of the $\mathrm{M}$ remain, where $\epsilon \equiv k_{P P} / k_{M M} \ll 1$.

Returning to the present case, if we assume all radical groups have similar reactivities $Q \approx t_{a}^{-1}$ we identify $T \approx\left(\lambda n_{0}\right)^{-1}=t_{m}^{*}$. (This is the MF timescale we have met previously, as expected: small molecules in $d=3$ obey MF kinetics.) Let us suppose $n_{0}$ is chosen so the polymer radicals are strongly overlapping, $n_{0} R^{3} \gg 1$ as in fig. 2. The conclusion is that after $t_{m}^{*}$ our reactive polymer system belongs to the $\mathrm{S}^{\text {conc }}$ region [30] since $Q$ exceeds the $Q^{*}$ threshold. But in region $\mathrm{S}^{\text {conc }}$ by definition $t_{m}^{*}$ is much less than the time $t_{l}$ for a reactive polymer end group to diffuse the distance between reactive ends. Thus (consistent with the above arguments) macroradicals cannot have substantially reacted with one another by time $t_{m}^{*}$. Monitoring the decay of macroradical density for $t>t_{m}^{*}$, the full sequence of kinetics is thus experimentally accessible, i. e. $n_{t} \approx n_{0}-n_{0}^{2} x_{t}^{3}$ for $t_{m}^{*}<t<t_{l}$ followed by $n_{t} \approx 1 / x_{t}^{3}$ for $t_{l}<t<\tau$. Experiments of this type thus hold promise for the measurement of fundamental scaling laws of polymer-polymer reaction kinetics.

This work was supported by the National Science Foundation under grant no. DMR9403566. 


\section{Appendix A. Derivation of eq. (5)}

Eq. (4) can be written equivalently as 18

$\frac{\partial \rho_{t}\left(\mathbf{r}_{1}, \mathbf{r}_{2}\right)}{\partial t}-\mathcal{D} \rho_{t}\left(\mathbf{r}_{1}, \mathbf{r}_{2}\right)=-\lambda \rho_{t}\left(\mathbf{r}_{1}, \mathbf{r}_{2}\right) \delta\left(\mathbf{r}_{1}-\mathbf{r}_{2}\right)-\lambda \int d \mathbf{r}_{3} \rho_{t}^{(3)}\left(\mathbf{r}_{1}, \mathbf{r}_{2}, \mathbf{r}_{3}\right)\left\{\delta\left(\mathbf{r}_{1}-\mathbf{r}_{3}\right)+\delta\left(\mathbf{r}_{2}-\mathbf{r}_{3}\right)\right\}$

where the terms on the right hand side represent reaction sinks and $\mathcal{D}$ is the diffusion operator. The propagator $G$ in eq. (4) is the inverse of $(\partial / \partial t-\mathcal{D})$. Notice that $-\mathcal{D} \rho$ is equal to the divergence of the reactive chain end current $\mathbf{j}$ at $\mathbf{r}_{1}, \mathbf{r}_{2}: \mathcal{D} \rho=-\left(\nabla_{\mathbf{r}_{1}}+\nabla_{\mathbf{r}_{2}}\right) \mathbf{j}\left(\mathbf{r}_{1}, \mathbf{r}_{2}\right)$. (For example for small molecules one would have $\mathbf{j}=-D\left(\nabla_{\mathbf{r}_{1}}+\nabla_{\mathbf{r}_{2}}\right) \rho$, where $D$ is the diffusion coefficient.) Since $\mathbf{j}$ must be linear in $\rho$, one has $\mathcal{D} \rho_{t}=\mathcal{D} q_{t} n_{t}^{2}=n_{t}^{2} \mathcal{D} q_{t}$. Then, substituting $\rho_{t}\left(\mathbf{r}_{1}, \mathbf{r}_{2}\right)=q_{t}\left(\mathbf{r}_{1}, \mathbf{r}_{2}\right) n_{t}^{2}$ in eq. (A1) one obtains

$\frac{\partial q_{t}\left(\mathbf{r}_{1}, \mathbf{r}_{2}\right)}{\partial t}-\mathcal{D} q_{t}\left(\mathbf{r}_{1}, \mathbf{r}_{2}\right)=-\lambda q_{t}\left(\mathbf{r}_{1}, \mathbf{r}_{2}\right) \delta\left(\mathbf{r}_{1}-\mathbf{r}_{2}\right)+2 \rho_{t}\left(\mathbf{r}_{1}-\mathbf{r}_{2}, 0\right) k_{t} n_{t}-2 \rho_{t}^{(3)}\left(\mathbf{r}_{1}-\mathbf{r}_{2}, 0,0\right) / n_{t}^{2}$

where we have performed the $\mathbf{r}_{3}$ integration in eq. (A1) and used the identities $\rho_{t}\left(\mathbf{r}_{1}, \mathbf{r}_{2}\right)=$ $\rho_{t}\left(\mathbf{r}_{1}-\mathbf{r}_{2}, 0\right)$ and $\rho_{t}^{(3)}\left(\mathbf{r}_{1}, \mathbf{r}_{2}, \mathbf{r}_{1}\right)=\rho_{t}^{(3)}\left(\mathbf{r}_{1}, \mathbf{r}_{2}, \mathbf{r}_{2}\right)=\rho_{t}^{(3)}\left(\mathbf{r}_{1}-\mathbf{r}_{2}, 0,0\right)$ which result from translational invariance. Inverting eq. (A2) one obtains eq. (5) after using eqs. (11), (3) and the definitions of the conditional densities in eq. (6).

\section{Appendix B. Bounds on $\varphi_{t}(0)$}

This appendix shows that the deletion of the positive term $\varphi_{t}(0)$, which results in eq. (7) resulting in eq. (12) gives the correct scaling solution for $k_{t}$. We show this by first determining an upper bound, namely $\varphi_{t}^{\max }(0)$, by finding the function $\mu_{t^{\prime}}^{\max }\left(\mathbf{r}^{\prime}\right)$ in the definition of $\varphi$ ( eq. (8)), which subject to the constraints satisfied by the real $\mu$, eqs. (10) and (11), maximizes $\varphi_{t}(0)$. We consider each of the 4 regions in figs. 4, 5 in turn.

\section{Region $\mathbf{S}^{\text {conc }}$}

$A$. Times $t \ll t_{l}$. During these times $n_{t} \approx n_{0}$ to within higher order corrections (see eq. (19)). In this case we just need to consider the constraint imposed on $\mu$ by eq. (10) only (including constraint (11) would lead to an even more strict bound which is not necessary here). Thus eq. (10) implies $\mu_{t^{\prime}}^{\max }\left(\mathbf{r}^{\prime}\right)=2 n_{t}$. Substituting $\mu^{\max }$ in eq. (8) and performing the $\mathbf{r}^{\prime}$ integration leads to

$$
\varphi_{t}^{\max }(0)=n_{0} \int_{0}^{t} d t^{\prime} k_{t}^{\prime} \approx \begin{cases}t / t_{m}^{*} & \left(t \ll t_{2}^{*}\right) \\ n_{0} x_{t}^{3} & \left(t_{2}^{*} \ll t \ll t_{l}\right)\end{cases}
$$

after use of eq. (15). In the $S^{\text {conc }}$ regime $t_{2}^{*} \ll t_{m}^{*}$ and $t \ll t_{l}$. Thus eq. (B1) implies $\varphi_{t}^{\max }(0) \ll 1$. Hence during this regime $\varphi$ is unimportant since it is much smaller than terms 1 and $q_{t}(0,0)$ in eq. (17). Eq. (12) is thus expected to give the correct $k$ (even the prefactor will not be modified by deletion of $\varphi$ ).

B. Times $t_{l} \ll t \ll \tau$. In this case we consider both constraints (10) and (11). Now clearly from its definition in eq. (8), $\varphi_{t}(0)$ is maximized when the integral of $k\left(t^{\prime}\right) \mu_{t^{\prime}}\left(\mathbf{r}^{\prime}\right)$ 
has its maximum value; this corresponds to replacing the inequality in (11) by an equality. Moreover, $\mu_{t^{\prime}}\left(\mathbf{r}^{\prime}\right)$ should be distributed around the points at which $G_{t-t^{\prime}}^{\text {sep }}\left(0, \mathbf{r}^{\prime}\right)$ in the definition of $\varphi_{t}(0)$ in eq. (8) is maximum. But $G^{\text {sep }}$ has the well known scaling form

$$
G_{t}^{\mathrm{sep}}(0, \mathbf{r}) \approx \begin{cases}1 / x_{t}^{3} & \left(r<x_{t}\right) \\ 0 & \left(r>x_{t}\right)\end{cases}
$$

Thus $G_{t-t^{\prime}}^{\text {sep }}\left(0, \mathbf{r}^{\prime}\right)$ is roughly constant (in space) for $r^{\prime}<x_{t-t^{\prime}}$ and vanishes for larger $r^{\prime}$. For a given $r^{\prime}$, it achieves maximum values at the maximum available $t^{\prime}$ values outside the vanishing region. Furthermore, we note that the maximum amplitude of $\mu_{t^{\prime}}^{\max }$ is of order $n\left(t^{\prime}\right)$. Hence the function maximizing $\varphi_{t}(0)$ is approximately

$\mu_{t^{\prime}}^{\max }\left(\mathbf{r}^{\prime}\right) \approx\left\{\begin{array}{ll}n\left(t^{\prime}\right) \Theta\left(x_{t-t^{\prime}}-r^{\prime}\right) & \left(t_{\mathrm{c}}<t^{\prime}<t\right) \\ 0 & \left(t^{\prime}<t_{\mathrm{c}}\right)\end{array}, \quad \int_{0}^{t} k\left(t^{\prime}\right) d t^{\prime}=\int_{t_{\mathrm{c}}}^{t} d t^{\prime} \int d \mathbf{r}^{\prime} k\left(t^{\prime}\right) \mu_{t^{\prime}}^{\max }\left(\mathbf{r}^{\prime}\right)\right.$,

where $\Theta$ is the step function $(\Theta(x)=0$ for $x<0, \Theta(x)=1$ for $x \geq 0)$. That is, $\mu_{t^{\prime}}^{\max }\left(\mathbf{r}^{\prime}\right)$ is localized at $\left(t^{\prime}, \mathbf{r}^{\prime}\right)$ close to $(t, 0)$ where it has its maximum amplitude. The time $t_{\mathrm{c}}$ defines the lower limit of the support of $\mu^{\max }$ and is determined by demanding that the total integral of $k \mu^{\max }$ yields the required value. Assuming that $t_{\mathrm{c}}$ is of order $t$ (which is verified self-consistently), we determine $t_{\mathrm{c}}$ by substituting $k_{t}$ and $n_{t}$ from eqs. (15) and (19) in eq. (B33) and performing the $\mathbf{r}^{\prime}$ integration. It is simple to show that this implies $\int_{t_{c} / t}^{1}(1-u)^{3 / z} / u d u \approx 1$, after using $x_{t} \sim t^{1 / z}$. Hence $t_{\mathrm{c}}=\beta t$, where $\beta$ is a constant of order unity $(0<\beta<1)$.

Substituting $\mu^{\max }$ into the expression for $\varphi_{t}(0)$ in eq. (8) and integrating over $\mathbf{r}^{\prime}$ one obtains $\varphi_{t}^{\max }(0) \approx \int_{t_{\mathrm{c}}}^{t} d t^{\prime} k\left(t^{\prime}\right) n\left(t^{\prime}\right) \approx \ln \left[n\left(t_{\mathrm{c}}\right) / n(t)\right]$ after use of eq. (四). Since $t_{\mathrm{c}}=\beta t$ thus $\varphi_{t}^{\max }(0)=A$, where $A$ is a constant of order unity.

We now argue that, assuming $k_{t}$ is still a power law, and given the boundndess of $\varphi_{t}(0)$, this implies $k_{t} \approx x_{t}^{3} / t$ during this regime. Substituting $k_{t} \sim t^{-\alpha}$ in the integral term in eq. (7) and setting $\mathbf{r}=0$, one easily derives the time-dependence of this term is $t^{\alpha-(3-z) / z}$. Since $\varphi_{t}(0)$ positive and bounded by $A$, and since the term $q_{t}(0,0)$ tends to 0 at long times, the only way to satisfy eq. (7) for $\mathbf{r}=0$ is by setting $\alpha=(3-z) / z$. This implies $k_{t} \approx x_{t}^{3} / t$. For this value of $\alpha$ the integral term in eq. (7) tends to a constant. Hence if $\varphi_{t}(0)$ is of order unity during this regime, it must be a constant in order for eq. (7) to be satisfied. The actual numerical prefactor of $k_{t}$ will then be a function of the value of $\varphi_{t}(0)$.

$C$. Times $t \gg \tau$. During these times $n_{t} \approx 1 /\left(R^{3} t / \tau\right)$ and $k_{t} \approx R^{3} / \tau$ (see eqs. (19) and (15)). We use the same reasoning as in part 1.B. up to eq. (B3). As in 1.B., it is easy to show that $t_{\mathrm{c}} / t=1-\gamma$, where $\gamma \approx\left[R^{3} t /\left(x_{t}^{3} \tau\right)\right]^{2 / 5}$ and that $\varphi_{t}^{\max }(0) \approx \gamma$. Since $\gamma \ll 1$ for $t \gg \tau$, it follows that $\varphi_{t}(0) \ll 1$ and that $\varphi_{t}(0)$ may be deleted from eq. (17).

\section{Region $\mathrm{W}$}

A. Times $t \ll t_{m}^{*}$. Similar arguments as in part 1.A. apply.

B. Times $t_{m}^{*} \ll t \ll t_{2}^{*}$. The procedure is the exactly the same as in part 1.C., but now using $k_{t} \approx \lambda$ and $n_{t} \approx 1 / \lambda t$ (see eqs. (15) and (20)). In this case one has $t_{\mathrm{c}} / t=1-\gamma$ with $\gamma \approx\left(\lambda t / x_{t}^{3}\right)^{2 / 5}$ and $\varphi_{t}^{\max }(0) \approx \gamma$. Since $\gamma \ll 1$ for $t \ll t_{2}^{*}$ it follows that $\varphi_{t}(0) \ll 1$ during this regime.

C. Times $t \gg t_{2}^{*}$. Identical to 1.B. for $t_{2}^{*} \ll t \ll \tau$ and to part 1.C. for $t \gg \tau$. 


\section{Region $\mathbf{S}^{\text {dil }}$}

A. Times $t \ll t_{m, R}^{*}$. For times $t \ll \tau$, same as part 1 .A. During this regime $\varphi_{t}^{\max }(0)$ in eq. (B1) is much smaller than unity since $t_{l}>\tau$. For $\tau \ll t \ll t_{m, R}^{*}$, similarly to 1.A., one finds $\varphi_{t}^{\max }(0)=t / t_{m, R}^{*}$ which is much smaller than unity.

B. Times $t \gg t_{m, R}^{*}$. Same as part 1.C.

\section{Region WW}

A. Times $t \ll t_{m}^{*}$. Same as part 1.A.

$B$. Times $t \gg t_{m}^{*}$. Same as part 2.B., but now notice that $\gamma \ll 1$ for all $t$ ( $t_{2}^{*}$ is not defined in this region). 


\section{References}

[1] M. Doi, Chem. Phys. 11, 115-121 (1975).

[2] P. G. de Gennes, J. Chem. Phys. 76, 3316-3321, 3322-3326 (1982).

[3] B. Friedman and B. O'Shaughnessy, Europhys. Lett. 23, 667 (1993); Macromolecules, 26, 5726 (1993).

[4] P. Flory, Principles of Polymer Chemistry (Cornell University Press, Ithaca, New York, 1971).

[5] B. Friedman and B. O'Shaughnessy, Int. J. Mod. Phys. B 8, 2555-2591 (1994).

[6] I. Mita and K. Horie, J. Macromol. Sci., Rev. Macromol. Chem. Phys. C27(1), 91-169 (1987).

[7] M. D. Wisnudel and J. M. Torkelson, J. Polym. Sci., Polym. Phys. Ed. 34, 2999 (1996).

[8] G. S. Grest, K. Kremer, and E. R. Duering, Europhys. Lett. 19, 195 (1992).

[9] G. S. Grest, K. Kremer, and E. R. Duering, Physica A 194, 330 (1993).

[10] E. Karatekin, B. O’Shaughnessy, and N. J. Turro, J. Chem. Phys. 108, 9577-9585 (1998).

[11] N. J. Turro, Modern Molecular Photochemistry (University Science Books, Mill Valley, California, 1991).

[12] K. Kang and S. Redner, Phys. Rev. A 32, 435 (1985).

[13] M. von Smoluchowski, Z. Phys. Chem. 92, 192 (1917).

[14] P. G. de Gennes, Scaling Concepts in Polymer Physics (Cornell Univ. Press, Ithaca, New York, 1985).

[15] M. Doi and S. F. Edwards, The Theory of Polymer Dynamics (Clarendon Press, Oxford, 1986).

[16] M. Doi, Chem. Phys. 11, 107-113 (1975).

[17] G. Wilemski and M. Fixman, J. Chem. Phys. 58, 4009 (1973).

[18] M. Doi, J. Phys. A 9, 1479 (1976).

[19] B. P. Lee, J. Phys. A 27, 2633-2652 (1994).

[20] B. P. Lee and J. Cardy, J. Stat. Phys. 80, 971-1007 (1995).

[21] E. Kotomin and V. Kuzovkov, Modern Aspects of Diffusion-Controlled Reactions; Cooperative phenomena in Bimolecular processes (Elsevier, Amsterdam, 1996), Vol. 34 of Comprehensive Chemical Kinetics. Edited by R. G. Compton and G. Hancock.

[22] E. T. Denisov, Liquid-Phase Reaction Rate Constants (IFI/Plenum, New York, 1974).

[23] Investigation of Rates and Mechanisms of Reactions, 4th ed., (John Wiley \& Sons, New York, 1986), Vol. VI of Techniques of Chemistry. Edited by Bernasconi C. L. 
[24] A. L. J. Beckwith, S. Brumby, R. F. Claridge, R. Crocket, and E. Roduner, in Radical Reaction Rates in Liquids, edited by H. Fischer (Springer-Verlag, Berlin, 1994), Vol. 18, Subvol. a of Landolt-Börnstein Numerical Data and Functional Relationships in Sciences and Technology. New Series. Group II, Atomic and Molecular Physics.

[25] B. O’Shaughnessy, Phys. Rev. Lett. 71, 3331 (1993); Macromolecules, 27, 3875, (1994).

[26] B. O'Shaughnessy. Bulk and Interfacial Polymer Reaction Kinetics. In Theoretical and Mathematical Models in Polymer Science, chapter 5, pages 219-275, Academic Press, New York, 1998. Edited by A. Grosberg.

[27] G. Oshanin, M. Moreau, and S. Burlatsky, Adv. Colloid Interface Sci. 49, 1 (1994).

[28] E. Karatekin, B. O'Shaughnessy, and N. J. Turro (Macromolecules, 1998, in press).

[29] B. O’Shaughnessy, Macromol. Theory Simul. 4, 481-496 (1995).

[30] A slight complication here is that during the transient $0<t<t_{m}^{*}$ the polymer-polymer reaction rate $k_{P P}(t)$ is time-dependent. However, the conclusions are essentially unchanged: the important feature is that $k_{P P}(t) \ll k_{M M}$ for all times $t \gg t_{a}$. 


\section{Figures}

FIG. 1. End-functionalized polymer chains (coil radius $R$ ) dispersed within a polymer melt of inert but otherwise identical chains. The initial density of reactive groups is $n_{0}$.

FIG. 2. Schematic of the situation immediately after photolysis of functional end-groups by a laser pulse generating radical pairs (consisting of a monomeric radical and a macroradical). The concentrated regime (overlapping reactive coils) is illustrated. After a transient most of the more mobile monomeric radicals react leaving behind a fraction of order unity of the polymer radicals. The situation of fig. 11 is then recovered.

FIG. 3. The depletion in the number density of reactive groups at $\mathbf{r}_{1}, \mathbf{r}_{2}$ originates from two terms in eq. (4). The two-body term subtracts off those pairs which would have been at $\mathbf{r}_{1}, \mathbf{r}_{2}$ at time $t$, but failed to do so because both members reacted at an earlier time $t^{\prime}$ at point $\mathbf{r}_{1}^{\prime}$. The many-body term subtracts off pairs which would have been at $\mathbf{r}_{1}, \mathbf{r}_{2}$ had there been no reactions, but failed to arrive because one member of the pair reacted at an earlier time.

FIG. 4. (a) Unentangled melts, reaction kinetics "phase diagram" in $Q-n_{0}$ plane. Axes are logarithmic and units chosen such that $t_{a}=a=1$. Maximum possible density is $n_{0} a^{3}=1 / N$ (all chains functionalized). Different sequences of kinetic behavior arise in the 4 regions $\mathrm{S}^{\text {conc }}, \mathrm{S}^{\text {dil }}, \mathrm{W}$ and WW. (b) As (a), but reactivities and densities expressed in terms of degree of polymerization $\mathrm{N}$.

FIG. 5. As fig. ॠ, but for entangled melts. Regions $\mathrm{S}^{\text {conc }}, \mathrm{S}^{\text {dil }}$ and $\mathrm{W}$ now develop fine

structure. In a given sub-region each relevant timescale occurs within a given reptation diffusion regime thus defining a unique sequence of kinetic regimes. 


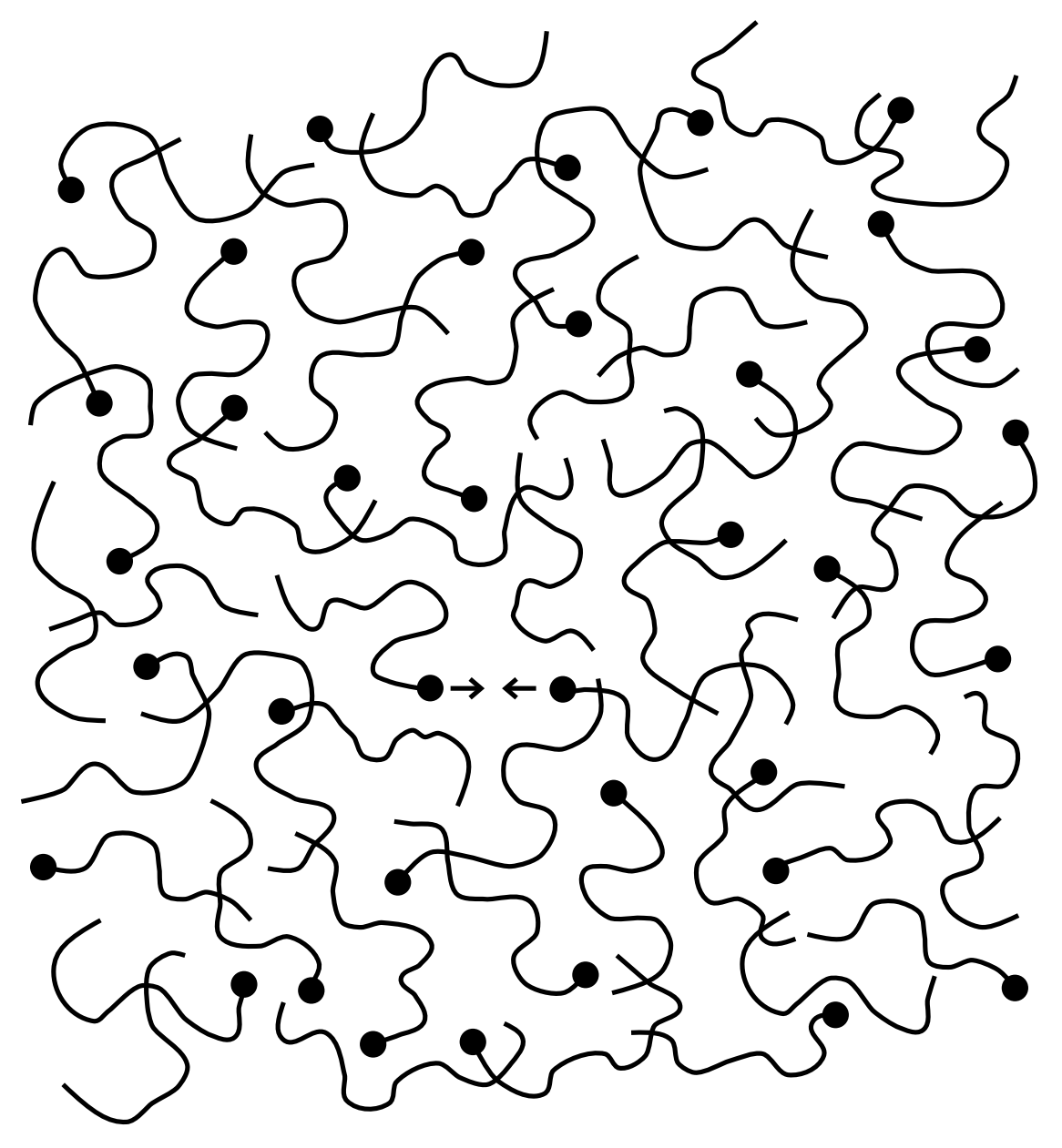

Fig. 1 


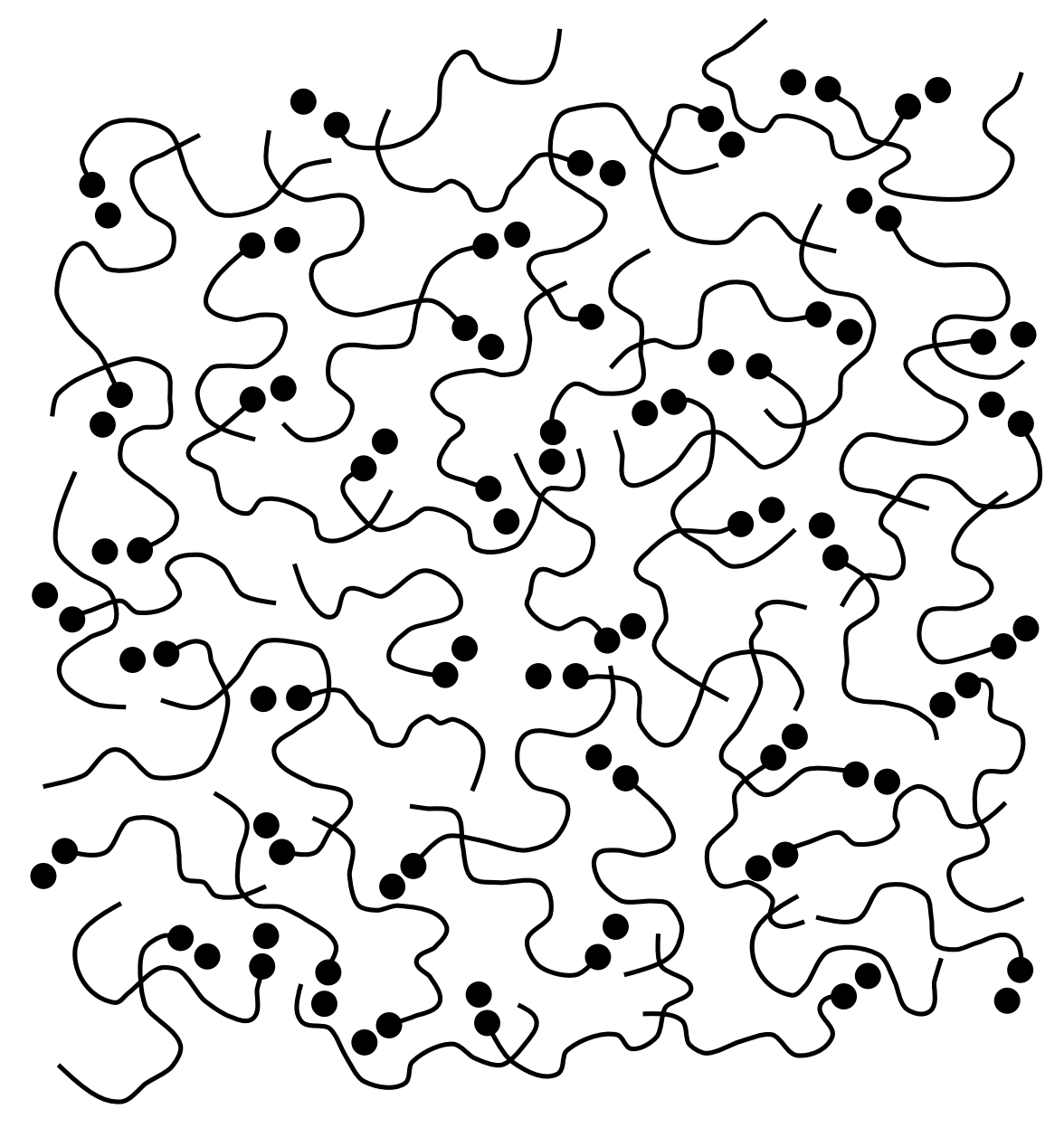

Fig. 2 
two-body term

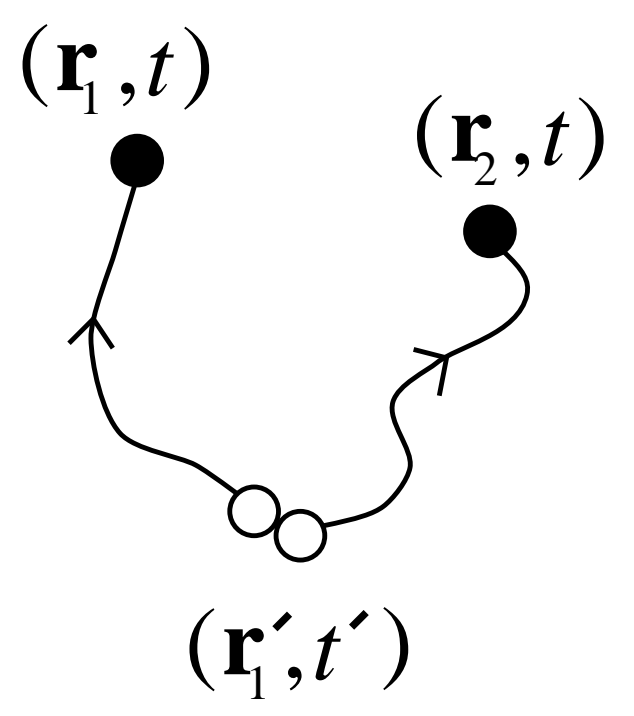

many-body term

$\left(\mathbf{r}_{1}, t\right)$

$\left(\mathbf{r}_{2}, t\right)$

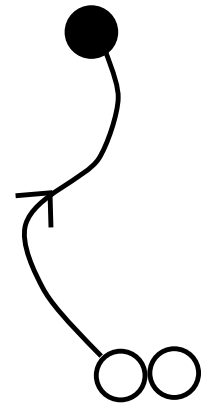

$\left(\mathbf{r}_{1}^{\prime}, t^{\prime}\right)$

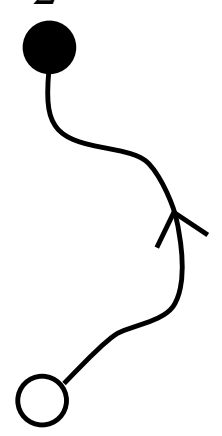

$\left(\mathbf{r}_{2}^{\prime}, t^{\prime}\right)$

Fig. 3 


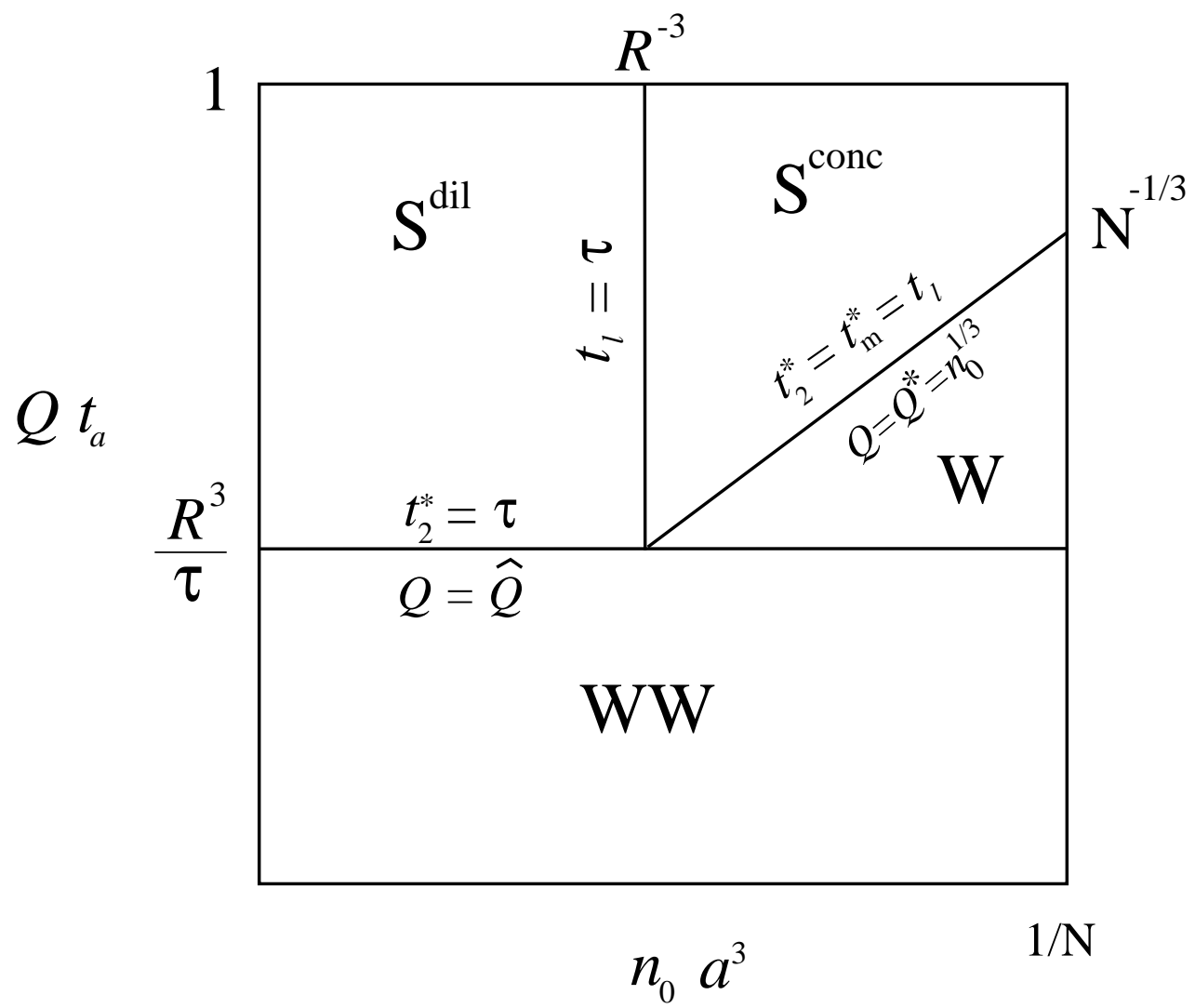

(a)

Fig. 4(a) 


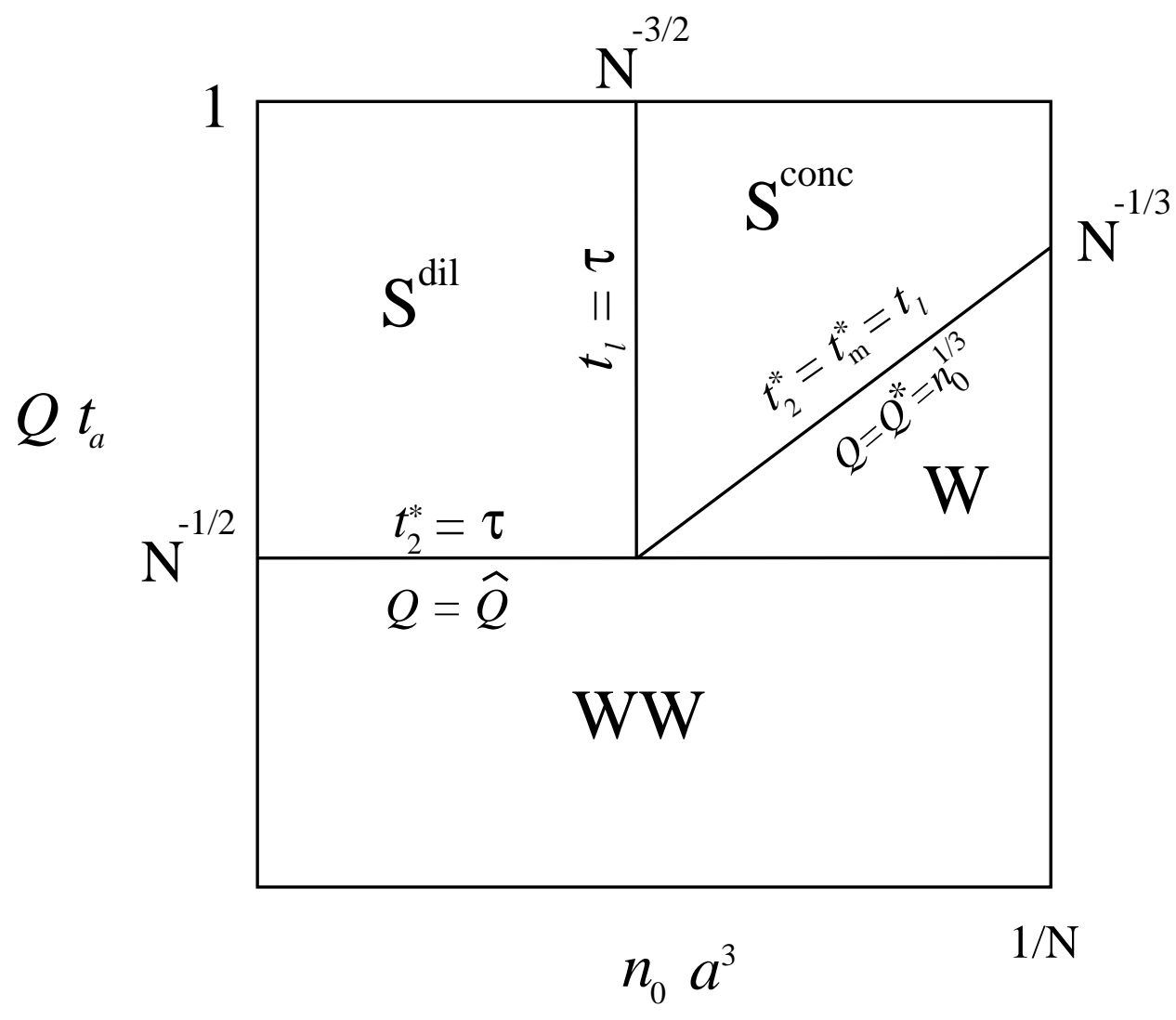

(b)

Fig. 4(b) 


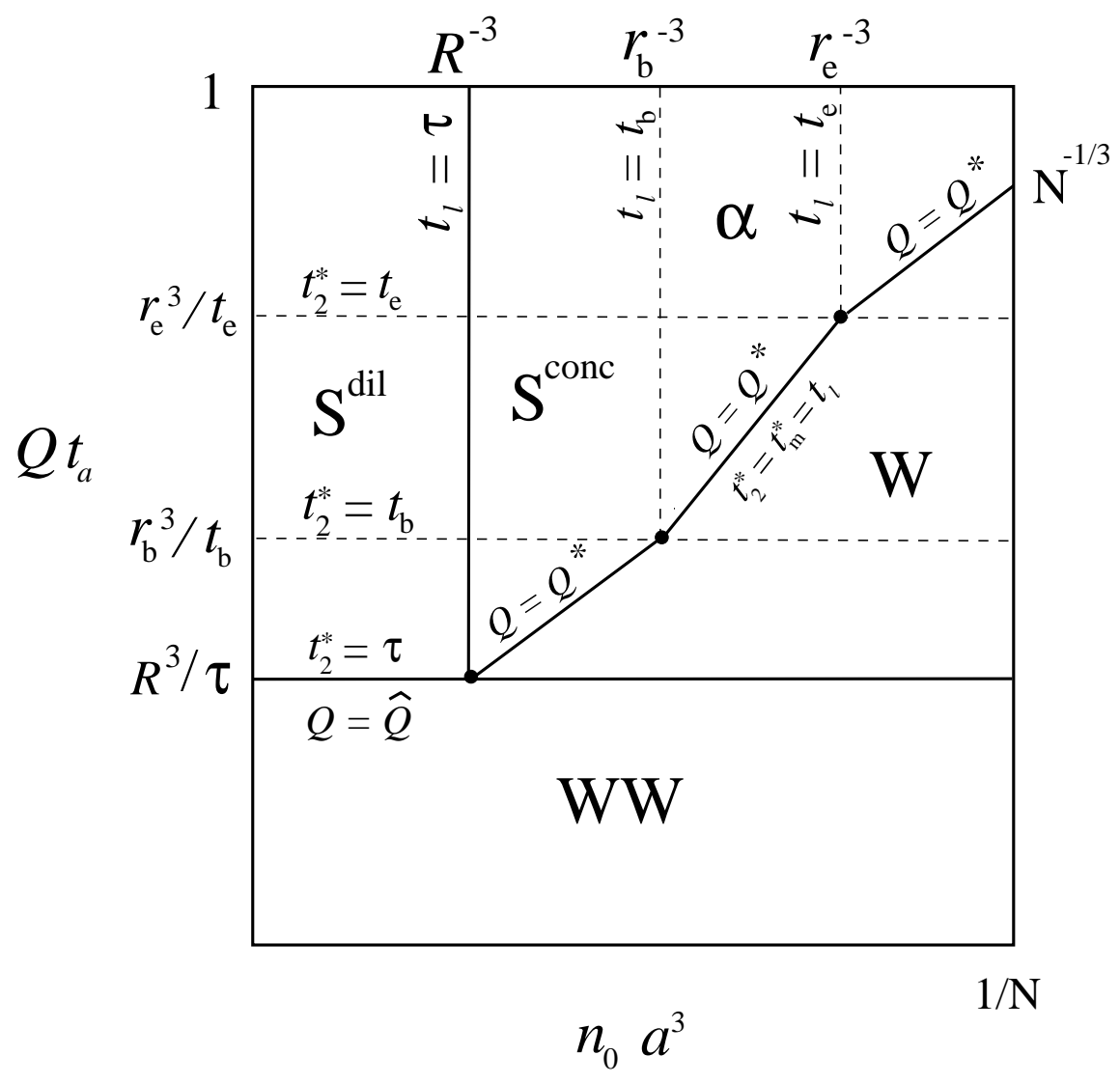

(a)

Fig. 5(a) 


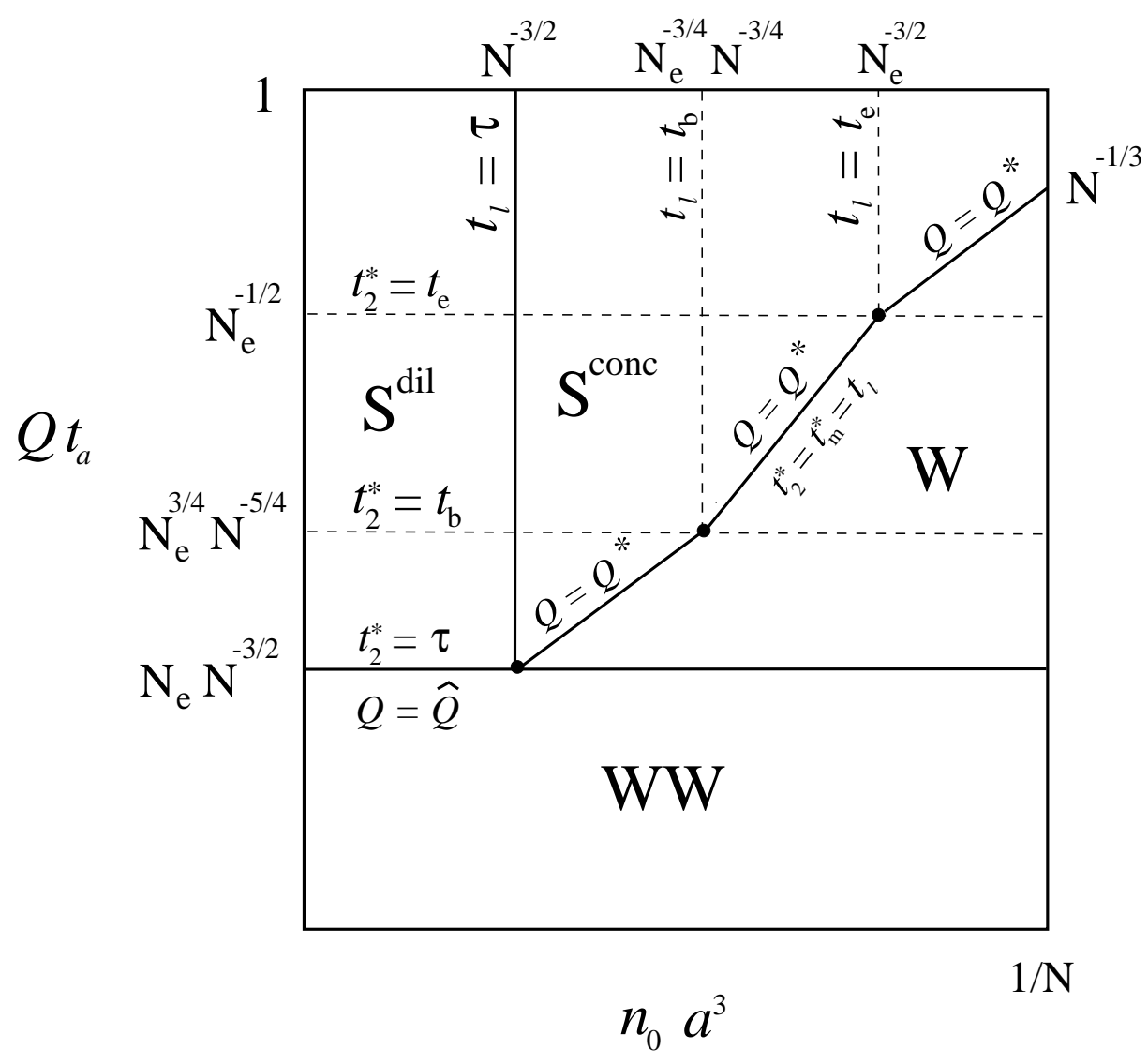

(b)

Fig. 5(b) 